\title{
ENTERIC \& 3D-PRINTED HYBRID PACKAGE FOR SAMPLING IN DIGESTIVE REGIONS
}

\author{
G.E. Banis ${ }^{1,3}$, L.A. Beardslee ${ }^{l}$, J.M. Stine ${ }^{1,2}$, and R. Ghodssi ${ }^{1,2}$ \\ ${ }^{1}$ Institute for Systems Research, ${ }^{2}$ Department of Electrical and Computer Engineering, \\ ${ }^{3}$ Fischell Department of Bioengineering, \\ University of Maryland, College Park, Maryland 20742 USA
}

\section{ABSTRACT}

We present a packaging concept for an ingestible sensing capsule, which utilizes biodegradable polymers to target the sensors to specific areas of the gut. Interdigitated electrodes (IDE) are inserted into 3D-printed capsules with embedded gratings that are filled with pharmaceutical polymers tuned to dissolve at a specific $\mathrm{pH}$ threshold. Sensors, housed within a 3D-printed package accessible only by the gratings, are connected to a microcontroller capable of transmitting data to a smartphone via Bluetooth Low-Energy (BLE). The system is tested with varying amounts and types of enteric coatings over the gratings, used toward optimizing control over timed polymer dissolution. This represents a minimally-invasive strategy toward in situ sampling and analysis at $\mathrm{pH}$-targeted locations in the gastrointestinal (GI) tract.

\section{INTRODUCTION}

Integrated capsule systems are widely gaining momentum for analysis or drug delivery in GI regions where traditional techniques are expensive, highly invasive, or inadequate [1], [2]. Depending on the region, there are numerous physiological considerations that must be addressed for fluid sampling and sensing with minimal human intervention [3]. Previous passive approaches to GI sampling lack a means of knowing whether a sample has been retrieved in real-time, while active approaches suffer due to cost and complex assembly [4]. In this work, a breadboard level circuit representative of the electronics, ultimately for integration within the device, is used to monitor the removal of polymer material from grids within a $3 \mathrm{D}$ printed capsule. The goal is to use the polymers to protect sensors within the capsule, only exposing them when the device has reached the GI region of interest [5]. Different Eudragit polymers are used to target different GI regions, depicted in Figure 1: E PO, S 100, and L 100 dissolve respectively at the stomach, ileum, and duodenum.

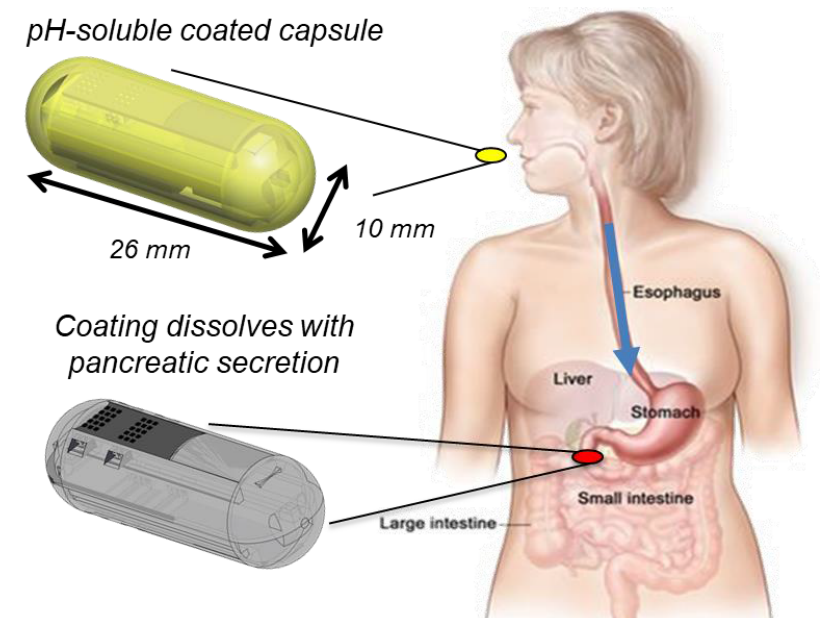

Figure 1: Depiction of system outlook and application (Image credits: NIH). Polymer coatings are intended to dissolve at either the stomach ( $p H$ 1.5-3) or the duodenum ( $p H$ 7-8.5). At the duodenum, gastric acid-neutralizing bicarbonate is secreted from the pancreas via the sphincter of Oddi.

978-1-940470-03-0/HH2018/\$25@2018TRF

DOI 10.31438/trf.hh2018.27

\section{WORKING PRINCIPLE AND RESULTS}

Figure 2 presents a 3D representation of the capsule (left) and a schematic overview of the electronics. Each die, containing four sensors, is inserted into a slot designed in one end of the 3D-printed capsule. Once inserted, the capsule was fastened using built-in threads and sealed with epoxy, then dip-coated into a $30 \mathrm{w} / \mathrm{v} \%$ solution of each polymer in methanol, each for 1-5 coats with removal for $20 \mathrm{~min}$ intervals between each coating. The circuit setup is depicted in Figure 2, characterized in Figure 3a, and transmission protocols are compared in Figure $3 \mathrm{~b}$.

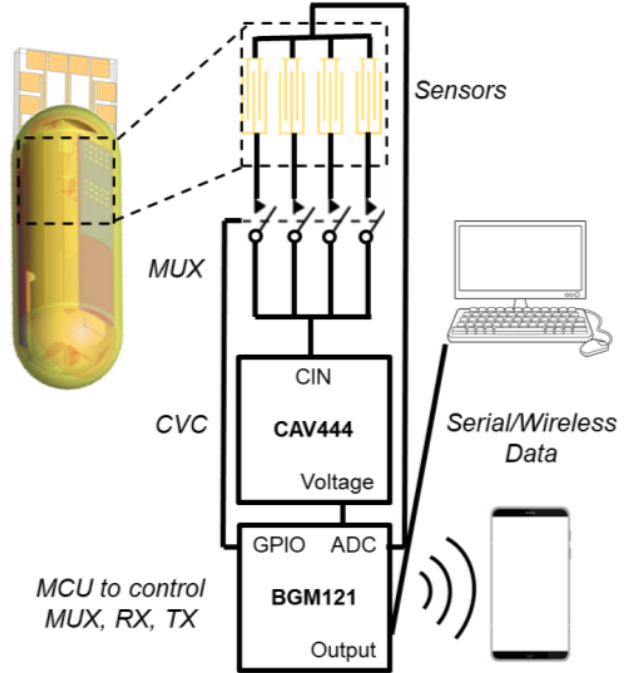

Figure 2: System overview, including the packaging design with the inserted IDEs and the circuit design. The capsule is made from MED610 using a Connex 3 Objet500 (Stratasys). Capacitor electrodes consisting of $\mathrm{Cr}(20 \mathrm{~nm}) / \mathrm{Au}(200 \mathrm{~nm}) \mathrm{IDEs}$ with $75 \mu \mathrm{m}$ width and spacing were fabricated using photolithography and lift-off over a pyrex wafer. Circuit design includes a multiplexer (MUX) integrated circuit (IC), a capacitance-voltage-converter (CVC) IC, a $1.5 \mathrm{MHz}$ step-up DC-DC converter, and a BLE System-in-Package (SIP) microcontroller unit (MCU).
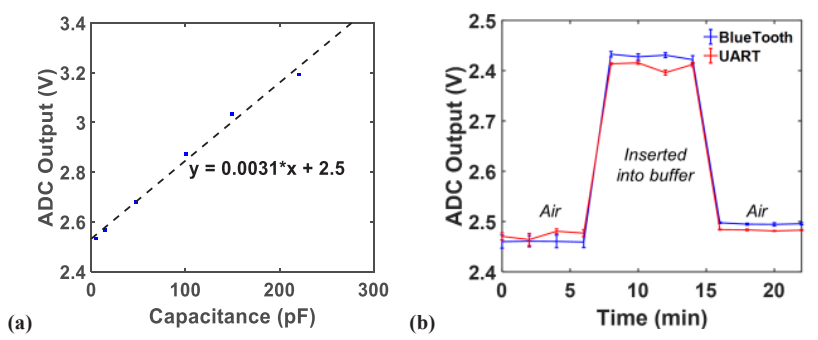

Figure 3: (a) Circuit calibration with standard capacitors across four multiplexed inputs. The resulting system senses capacitive changes in the $0.8-220 \mathrm{pF}$ dynamic range with a sensitivity of $3.2 \times 10^{-3} \mathrm{pF} / \mathrm{mV}$ and operated using a 3.3 V source. (b) Comparison of data obtained through Bluetooth versus serial (UART) communication, where the capsule (open gratings) was measured in air, inserted into buffer, then removed $(n=4)$. Hilton Head Island, South Carolina, June 3-7, 2018 
Capsule coating thicknesses for S 100 were analyzed, shown in Figure 4, over gratings and non-grating regions. Gratings resulted in a slower increase (Figure 4d) with each coating due to polymer infill. Capsules were then immersed in $0.1 \mathrm{M}$ acetic acid $(\mathrm{pH} 3)$ and progressively adjusted at $30 \mathrm{~min}$ intervals to increasing $\mathrm{pH}$ levels. Figure 5 presents the sensor output and corresponding change in capacitance over the course of $\mathrm{pH}$ adjustments for the $\mathrm{S} 100$ polymers, indicating the expected length of time for chamber filling and sampling dynamics for each coating thickness.
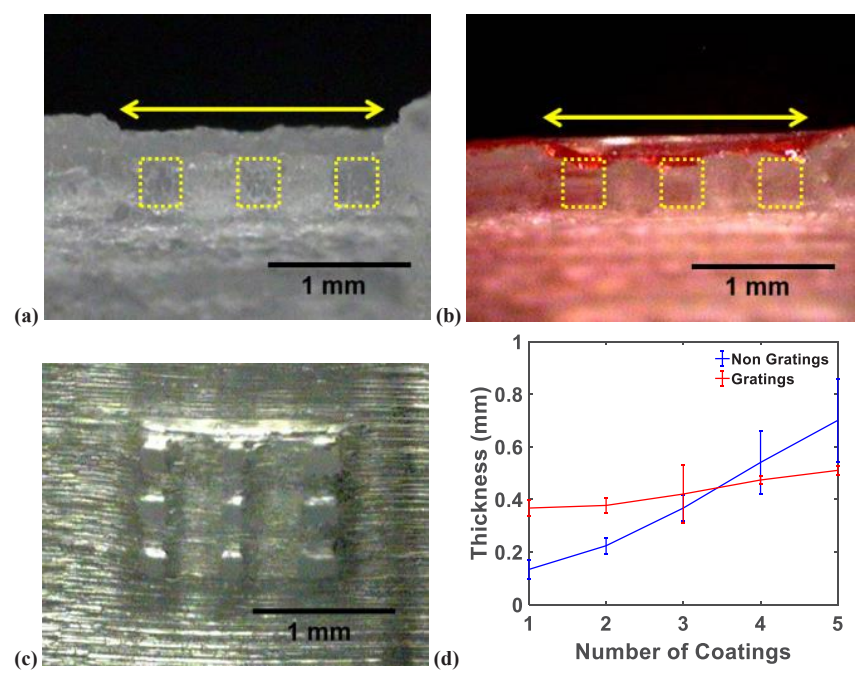

Figure 4: Cross-sectional optical images of the sampling gratings (a) uncoated and (b) with 1 coating of red-dyed polymer, where yellow markers indicate the grating locations. (c) Top-view optical image of uncoated gratings. (d) Characterization of polymer coating thickness vs. number of dipping steps from both non-grating and grating regions over the $3 D$-printed capsule surface $(n=3)$.

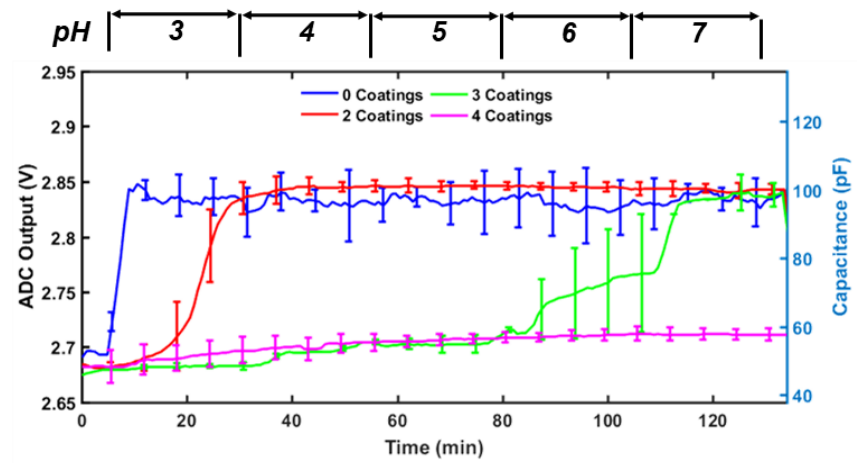

Figure 5: Sensor responses inserted (at $5 \mathrm{~min}$ ) into capsules with different numbers of $S 100$ coatings (0,2, 3, and 4, respectively) with increasing $\mathrm{pH}$ using titers of $1 \mathrm{M} \mathrm{NaOH}(n=4)$. Chamber filling is characterized by a capacitance change of $\sim 50 \mathrm{pF}$ from the initial capacitance in air. For each formulation, it was determined that 3 coatings was optimal for the $w / v \%$ used.

After coating optimization was performed for each polymer, combined coatings were tested to determine ability to tailor sampling for more complex $\mathrm{pH}$ sequences. Figure 6 presents representative sequences of L 100 and E PO formulations ( $a$ and b, respectively), as well as a combined coating of both polymers. As expected, the capacitance increase did not occur until $\sim \mathrm{pH} 5$, indicative that this combined coating strategy can be used to protect the sensing chambers until the characteristic $\mathrm{pH}$-targeted regions are reached.
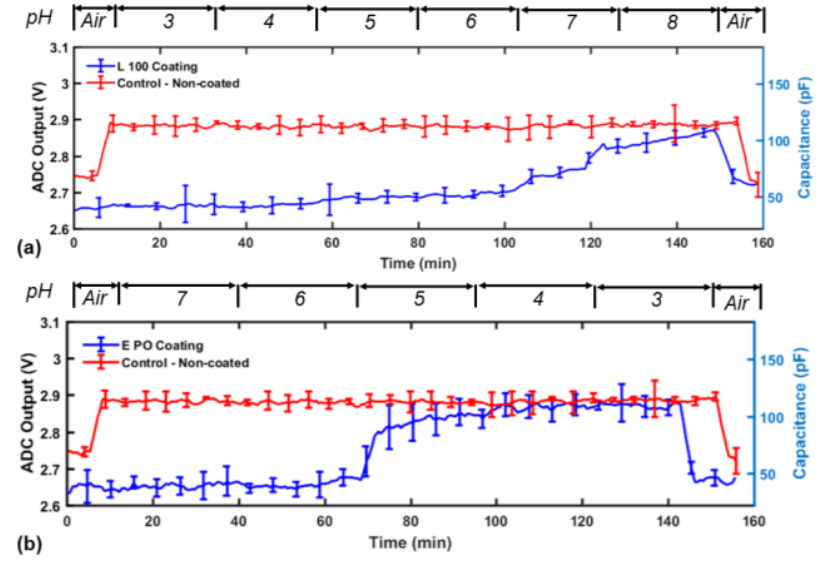

(b)

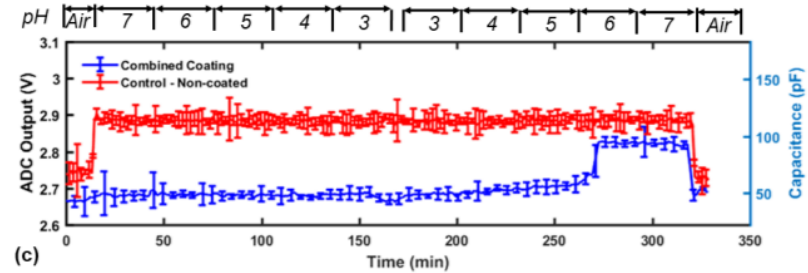

Figure 6: Sensor responses inserted into capsules with different types of coatings (3 dip-coatings each) compared to uncoated controls: (a) L 100, (b) E PO, and (c) combined coatings, where E PO is outer-most and $L 100$ is between the $3 D$-printed capsule and the E PO layers $(n=4)$.

\section{CONCLUSION}

This work is the first demonstration of a passive $\mathrm{pH}$-dependent packaging strategy for ingestible capsule technology that offers information on the package integrity at any given time, while simultaneously providing a real-time active microelectronics-based system capable of wireless retrieval of sensor data for use in GI sampling and analysis.

\section{ACKNOWLEDGEMENT}

This work was supported by the National Science Foundation ECCS Program under Award 1738211. The authors acknowledge the support from the Maryland NanoCenter and its FabLab.

\section{REFERENCES}

[1] J. Z. Ou, C. K. Yao, A. Rotbart, J. G. Muir, P. R. Gibson, and K. Kalantar-zadeh, "Human intestinal gas measurement systems: in vitro fermentation and gas capsules," Trends Biotechnol., vol. 33, no. 4, pp. 208-213, Apr. 2015.

[2] S. N. Adler and Y. C. Metzger, "PillCam COLON capsule endoscopy: recent advances and new insights," Ther. Adv. Gastroenterol., vol. 4, no. 4, pp. 265-268, Jul. 2011.

[3] G. Banis, A. L. Beardslee, and R. Ghodssi, "Gelatin-Enabled Microsensor for Pancreatic Trypsin Sensing," Appl. Sci., vol. 8 , no. 2, 2018.

[4] "Ingestible Gastrointestinal Sampling Devices: State-of-theArt and Future Directions," Crit. Rev. Biomed. Eng., vol. 42, no. 1, pp. 1-15, 2014.

[5] G. Banis, L. A. Beardslee, J. S. Stine, and R. Ghodssi, "PH Targeting via Packaging for a Wireless Ingestible Capsule," presented at The 9th International Conference on Microtechnologies in Medicine and Biology, Monterey, CA, Mar-2018.

\section{CONTACT}

*R. Ghodssi, tel: +1-301-405-8158; ghodssi@umd.com 